\title{
INDEX to Volume 65: BOOK REVIEWERS
}

Aguilar-Melendez, Araceli, 105

Anastassopoulos, Elias, 237

Applequist, Wendy, 227

Austin, Daniel F., 111, 112, 230

Bedigian, Dorothea, 343

Bonhage-Freund, Mary Theresa, 101, 336

Brendler, Thomas, 103, 226

Carty, Susan

Crone, Wilson, 235

Deb, Debal, 236

Drewal, Henry John, 228

Eich, Eckart

Emery, Homer C., 338

Felger, Richard, 110

Fisher, Jack B.
Johnson Fulton, Susannah B., 107, 108

Gilbert, Paul R., 234, 239

Giovannini, Peter, 226

Hall, Matthew, 228

Harriman, Neil A., 111, 230

Hodgson, Wendy C., 100

Ingram, Verina, 340

Janni, Kevin, 337

Katz, Esther, 233

Krueger, Robert J, 104

Larsen, Anna, 343

Lewis, Walter H., 335

Liao, Lawrence M., 341

Long, Marie

Lubinsky, Pesach, 232, 233
Macía, Manuel J., 342

Máthé, Ákos, 339

Miller, Chris, 105

Mishkin, Miramanni M.

Musselman, Lytton John

Offringa, Lisa, 102

Rashford, John H., 108

Redman, Nigel, 109

Siebert, Stephen F., 237

Stepp, John Richard, 338

Stewart, Kristine, 231

Storch, Diana, 106

Van Damme, Patrick

Vandebroek, Ina

Voeks, Robert, 100

Published online 24 February 2012. 